\title{
Non-invasive evaluation of nigrostriatal neuropathology in a proteasome inhibitor rodent model of Parkinson's disease
}

\author{
Anthony C Vernon, Saga M Johansson, Michel M Modo*
}

\begin{abstract}
Background: Predominantly, magnetic resonance imaging (MRI) studies in animal models of Parkinson's disease (PD) have focused on alterations in $\mathrm{T}_{2}$ water ${ }^{1} \mathrm{H}$ relaxation or ${ }^{1} \mathrm{H}$ MR spectroscopy (MRS), whilst potential morphological changes and their relationship to histological or behavioural outcomes have not been appropriately addressed. Therefore, in this study we have utilised MRI to scan in vivo brains from rodents bearing a nigrostriatal lesion induced by intranigral injection of the proteasome inhibitor lactacystin.

Results: Lactacystin induced parkinsonian-like behaviour, characterised by impaired contralateral forelimb grip strength and increased contralateral circling in response to apomorphine. $T_{2}$-weighted MRI, 3-weeks post-lesion, revealed significant morphological changes in PD-relevant brain areas, including the striatum and ventral midbrain in addition to a decrease in $\mathrm{T}_{2}$ water ${ }^{1} \mathrm{H}$ relaxation in the substantia nigra ( $\mathrm{SN}$ ), but not the striatum. Post-mortem histological analyses revealed extensive dopaminergic neuronal degeneration and $\alpha$-synuclein aggregation in the SN. However, extensive neuronal loss could also be observed in extra-nigral areas, suggesting non-specific toxicity of lactacystin. Iron accumulation could also be observed throughout the midbrain reflecting changes in $T_{2}$. Importantly, morphological, but not $\mathrm{T}_{2}$ relaxivity changes, were significantly associated with both behavioural and histological outcomes in this model.

Conclusions: A pattern of morphological changes in lactacystin-lesioned animals has been identified, as well as alterations in nigral $T_{2}$ relaxivity. The significant relationship of morphological changes with behavioural and histological outcomes in this model raises the possibility that these may be useful non-invasive surrogate markers of nigrostriatal degeneration in vivo.
\end{abstract}

\section{Background}

Parkinson's disease (PD) is a progressive neurodegenerative movement disorder characterised by a selective vulnerability and degeneration of dopaminergic (DA) neurons in the substantia nigra pars compacta (SNc) [1]. This is accompanied by formation of eosinophilic cytoplasmic inclusions in remaining neurons, termed Lewy bodies (LB), composed primarily of fibrillar aggregates of $\alpha$-synuclein [2].

Degeneration of DA neurons in the SNc results in significant depletion of striatal dopamine levels, which can be readily visualised in PD patients using Positron

\footnotetext{
* Correspondence: mike.modo@kcl.ac.uk Department of Neuroscience, Kings College London, Centre for the Cellular Basis of Behaviour, The James Black Centre, 125 Coldharbour Lane, London SE5 9NU, UK
}

(c) 2010 Vernon et al; licensee BioMed Central Ltd. This is an Open Access article distributed under the terms of the Creative Commons Attribution License (http://creativecommons.org/licenses/by/2.0), which permits unrestricted use, distribution, and reproduction in any medium, provided the original work is properly cited. fic radiotracers, such as 18 -fluorodopa $\left({ }^{18} \mathrm{~F}-\mathrm{DOPA}\right)$ [3]. However, whilst there is abundant PET data for PD, the results of magnetic resonance imaging (MRI)-morphometric studies regarding the basal ganglia (BG) in PD patients are still relatively scarce and inconsistent [4]. Indeed, brain atrophy is not routinely ascribed to human idiopathic PD (iPD), particularly in cognitively intact patients [5]. In early-stage iPD, both normal $[6,7]$ and partial reductions [8-10] in grey matter volume (GMV) of the BG have been reported [4]. Similarly, voxel-based morphometry (VBM) studies have reported significant morphological changes, including atrophy of the head of the left caudate nucleus and cortical changes in both early and advanced iPD, which correlated robustly with clinical symptoms $[4,11]$. Furthermore, 
significant asymmetrical hypertrophy of the lateral ventricles (LV) has been reported, which also correlated robustly with patient disability scores on the Unified Parkinson's Disease Rating Scale (UPDRS) [12]. Importantly, patterns of morphometric change which can be readily detected by MRI are already utilised clinically to aid differential diagnosis of iPD from other forms of parkinsonism, including the $\alpha$-synucleinopathy multiple system atrophy (MSA) and the tauopathy progressive supranuclear palsy (PSP) $[13,14]$.

In addition, it has been widely reported that $\mathrm{T}_{2}$ water proton relaxivity rates are decreased in pathologically relevant areas in iPD patients, including the SNc and putamen, which has been ascribed to regional iron accumulation [15-18]. Interestingly, a significant relationship between $\mathrm{T}_{2}$ relaxivity, iron accumulation and clinical symptoms has been described. Alterations in $\mathrm{T}_{2}$ relaxivity may therefore represent a surrogate marker for $\mathrm{PD}$ progression, although only cross-sectional studies have been performed to date $[19,20]$. By contrast, the possibility that morphometric changes, or a combination of this with alterations in $\mathrm{T}_{2}$ relaxation time may be used to non-invasively monitor disease progression in $\mathrm{PD}$ remains unclear [21].

Crucial to a more mechanistic understanding of pathology and the evaluation of novel treatments for PD are animal models that reflect important aspects of the clinical manifestation of the disease. Foremost of all, rodent toxin-based models of PD, although subject to limitations, have provided useful insights into the pathophysiology of iPD [22]. Combining such animal models with non-invasive imaging, such as MRI, offers a powerful tool with which to investigate potential dynamic morphological changes due to degeneration of the nigrostriatal system, which may lead to identification of surrogate markers of disease progression [23,24]. Importantly, detailed anatomical or relaxivity information can be correlated directly with behavioural phenotypes, raising the possibility that MRI could provide non-invasive surrogate markers predictive of the degree of functional impairment in individual animals [25].

Previous studies have elegantly demonstrated the application of MRI to animal models of PD [26-31]. Primarily however, these studies have focused on alterations in $\mathrm{T}_{2}$ relaxation or ${ }^{1} \mathrm{H}$ MR spectroscopy (MRS) and more recently diffusion tensor imaging (DTI) [32] and manganese enhanced MRI (MEMRI) [33]. In contrast, potential morphological changes and their relationship to histological or behavioural outcomes have not been appropriately addressed. Therefore, in this study we have utilised MRI to scan in vivo brains from rodents bearing a nigrostriatal lesion induced by an intranigral injection of the proteasome inhibitor lactacystin. This relatively recently developed model has been suggested to be a novel, pathologically relevant model of PD [34-36], which has already been used with some success in neuroprotection studies [35-38]. Our primary aim in this study was to investigate whether morphological and $\mathrm{T}_{2}$ relaxation times are altered in PD-relevant brain areas in this new experimental model of PD and to determine how these are related to behavioural and histological outcomes.

\section{Results \\ Behavioural testing}

Lactacystin-lesioned animals, but not controls, initially developed gross neurological deficits progressively, as shown by a gradual worsening in neurological score (Figure 1A). Lactacystin-lesioned animals consistently showed deficits in spontaneous motility, which may be a reflection of developing bradykinesia in these animals. Lesioned animals also demonstrated clear deficits in the horizontal bar, grasping reflex and placing reaction tests, suggesting defects in skilled forelimb use. Lesioned animals showed only moderate deficits in the cage top test and did not show any deficits in righting reflex or visual placement test (Figure 1B). Consistent with the initial progressive increase in global neurological score between 3 and 7 days, the percentage of lactacystinlesioned animals that displayed deficits in these tests increased in this period (Figure 1B). Modest further increases in the percentage of animals displaying these deficits were observed between days 7 to 21 post-lesion (Figure 1B). However, this likely reflects inter-animal variation in lesion size and not progressive lesion development, as global neurological scores remain static (Figure 1A,B). Qualitatively, lactacystin-lesioned animals, but not controls, also developed ipsiversive postural deficits and demonstrated spontaneous circling behaviour ipsilateral to the lesioned hemisphere. Importantly, neurological scores became static by 7 days post-lesion, and did not increase further until sacrifice 3 weeks postlesion (Figure 1A).

To determine if lactacystin-lesioning affects forelimb use, performance was assessed in the grip strength measure (GSM) test. Baseline measures of grip strength were established prior to surgery with no significant difference in average grip force observed for either forelimb in either group (Figure 1C,D). No significant deficits in grip strength between the contralateral and ipsilateral forelimbs were observed in saline-injected controls (Figure 1C). By contrast, 3-weeks post-surgery, lactacystin-lesioned animals displayed a significant decrease in the grip strength of the contralateral compared to the ipsilateral forelimb $(p<0.001$; Figure 1D). No significant differences in the grip strength of the ipsilateral limb were observed between lactacystinlesioned animals and controls at any time-point (Figure 


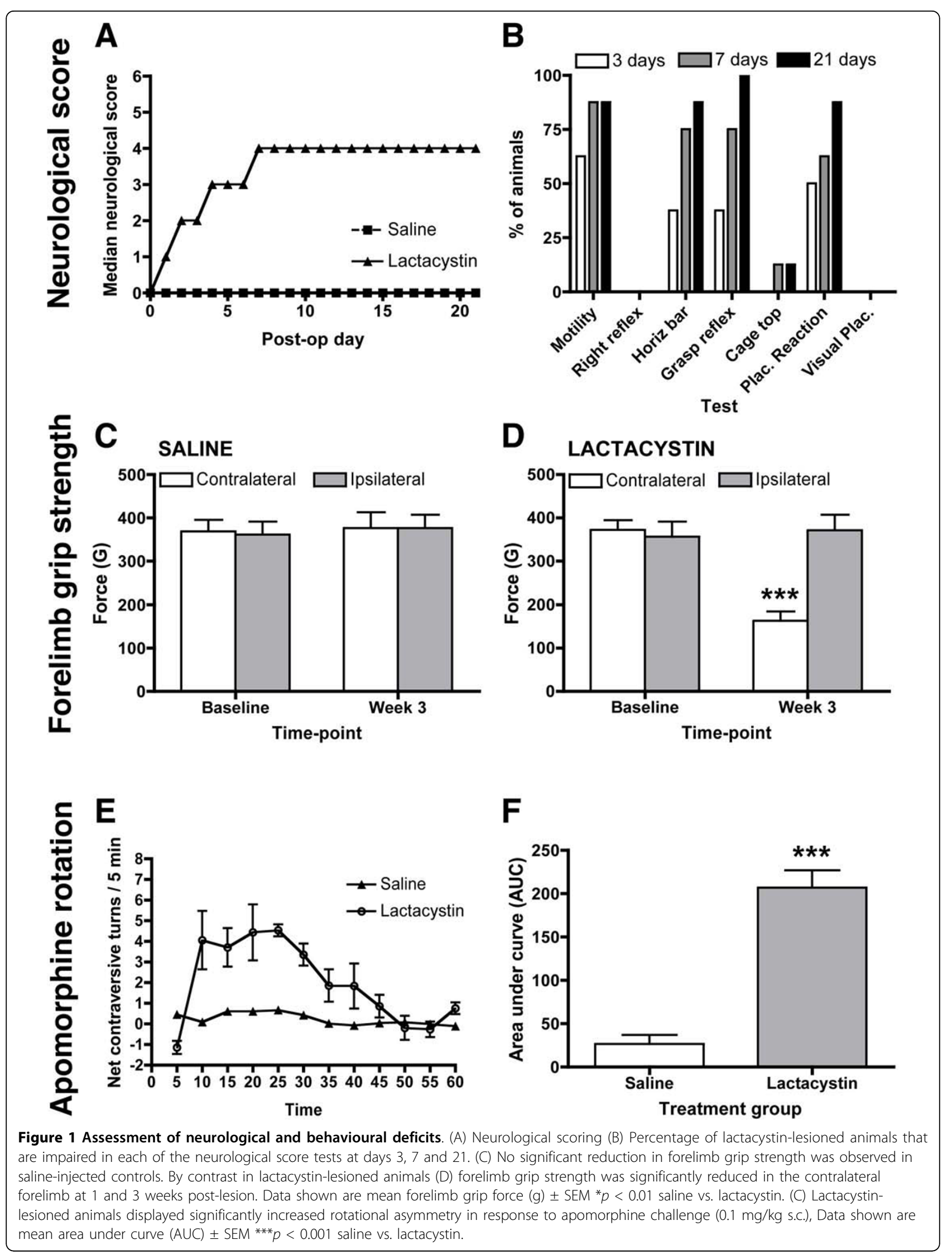


$1 C, D)$. Furthermore, three-weeks post-surgery, lactacystin-lesioned animals, but not saline controls, displayed significantly increased net contraversive rotations following an apomorphine challenge $(p<0.001$, Figure $1 \mathrm{E}, \mathrm{F})$. Lactacystin-lesioned animals displayed an average net contraversive turns of $>5$ turns/min. This activity increased steadily after $5 \mathrm{~min}$ and was maintained for approximately 35 minutes, at which point locomotor activity decreased back to baseline (Figure 1E). No spontaneous recovery of either neurological score or behaviour was observed during the 3 weeks of observation in lactacystin-lesioned animals.

\section{Brain regional volumes}

Sample ROI used to obtain volumetric data are shown in Figure 2A and the anatomical criteria used to define individual brain structures are shown in Table 1 . There was a significant reduction in whole brain volume (WBV) when comparing lactacystin-lesioned animals and saline controls $\left(1436 \pm 18\right.$ vs. $1375 \pm 11 \mathrm{~mm}^{3} ; p<$ 0.05 ; Figure 2B). Measurements of individual brain structures revealed numerous regional volume changes in lactacystin-lesioned animals, but not saline controls. Indeed, the ipsilateral striatum (STR) was found to be significantly smaller relative to the non-injected contralateral hemisphere in lactacystin-lesioned animals (20.04 \pm 0.93 vs. $24.93 \pm 0.40 \mathrm{~mm}^{3} ; p<0.001$; Figure $2 \mathrm{C}$ ) and the ipsilateral hemisphere of saline controls (20.04 \pm 0.40 vs. $26.70 \pm 0.79 \mathrm{~mm}^{3} ; p<0.001$; Figure 2C). In addition, the ipsilateral ventral midbrain (VM) was strikingly deformed with a concomitant increase in the surrounding cerebrospinal fluid (CSF) space. Quantitative measurement revealed the ipsilateral VM to be significantly smaller in lesioned animals compared to both the non-injected contralateral hemisphere $(38.03 \pm 0.69$ vs. $30.61 \pm 0.66 \mathrm{~mm}^{3} ; p<0.001$; Figure 2D) and the ipsilateral hemisphere of saline control animals (35.51 \pm 0.39 vs. $30.61 \pm 0.66 \mathrm{~mm}^{3} ; p<0.001$; Figure 2D).

Notably, the ipsilateral lateral ventricles (LV) were significantly enlarged in lactacystin-lesioned animals

Table 1 Anatomical criteria for volume measurements.

\begin{tabular}{ll}
\hline Brain region & Anatomical criteria for measurement \\
\hline $\begin{array}{l}\text { Whole Brain } \\
\text { Lateral }\end{array}$ & $\begin{array}{l}\text { Base of the olfactory bulb to last slice containing cortex } \\
\text { ventricles }\end{array}$ \\
$\begin{array}{ll}\text { Corpus } \\
\text { striatum }\end{array}$ & $\begin{array}{l}\text { Defined with reference to corpus callosum, external } \\
\text { capsule, anterior comissure and lateral ventricles }\end{array}$ \\
Midbrain & $\begin{array}{l}\text { Defined with reference to dorsal hippocampal } \\
\text { formation }\end{array}$ \\
Hippocampus & $\begin{array}{l}\text { Defined with reference to corpus callosum and external } \\
\text { capsule }\end{array}$ \\
Cerebellum & $\begin{array}{l}\text { Defined with reference to } 4^{\text {th }} \text { ventricle and brainstem } \\
\text { as a guide }\end{array}$ \\
\hline
\end{tabular}

$\overline{\text { Abbreviations: } \mathrm{AC}=\text { anterior commissure, } \mathrm{CC}=\text { corpus callosum, } \mathrm{EC}=\text { external }}$ capsule compared to both the non-injected contralateral hemisphere $\left(14.80 \pm 2.30\right.$ vs. $9.02 \pm 1.38 \mathrm{~mm}^{3} ; p<0.01$; Figure $2 \mathrm{E})$ and the ipsilateral hemisphere of control animals $\left(14.98 \pm 2.30\right.$ vs. $5.46 \pm 0.29 \mathrm{~mm}^{3} ; p<0.01$; Figure $2 \mathrm{E}$ ). The contralateral LV in lactacystin lesioned animals was also significantly enlarged compared to saline controls $\left(9.02 \pm 1.38\right.$ vs. $5.92 \pm 0.36 \mathrm{~mm}^{3} ; p<0.05$; Figure 2E).

No significant volume changes were observed in either the absolute volume of the cerebellum (CB) (Figure 2F) or for the hippocampal formation (HF) in either hemisphere, when comparing saline and lactacystin-injected animals (Figure 2F). These data are illustrated in representative MR images in Figure 3A, where clear hypertrophy of the LV can be observed (Figure 3A) as well as the striking deformation of the ipsilateral VM and corresponding increase in CSF space (Figure 3B). With the exception of the contralateral LV, no significant differences were observed for any brain region when comparing the contralateral hemisphere of saline and lactacystin-injected animals.

\section{$\mathrm{T}_{2}$ signal intensity measurements}

In lactacystin-lesioned animals, but not saline controls, visual inspection of $\mathrm{T} 2 \mathrm{~W}$ images revealed no visible areas of $\mathrm{T}_{2}$ signal change in the striatum in either hemisphere between saline and lactacystin-lesioned animals (Figure 3A). In contrast, areas of $\mathrm{T}_{2}$ hypointensity could be clearly observed in the VM, particularly in an area approximate to the location of the SN (Figure 3B). Notably, however, areas of $\mathrm{T}_{2}$ hypointensity were also observed in extra nigral regions throughout the ipsilateral VM (Figure 3B). These data are supported by quantitative ROI-based $\mathrm{T}_{2}$ measurements, which revealed no significant alterations in striatal $\mathrm{T}_{2}$ between hemispheres in either lactacystin-lesioned animals or saline controls (Figure 4A, B). By contrast, a moderate, but significant, decrease in $\mathrm{T}_{2}$ relaxivity was observed in the ipsilateral ventral midbrain and $\mathrm{SN}$ of lactacystin-lesioned animals, relative to the non-injected contralateral hemisphere and the ipsilateral SN of saline-injected controls (Figure 4C-F).

\section{Post-mortem histology}

To validate the origin of MRI signal changes and to examine how these are associated with nigrostriatal pathology, post-mortem immunohistological analyses were performed on the nigrostriatal system. Unbiased stereology cell counts revealed that intranigral injection of lactacystin resulted in an $81.4 \pm 1.1 \%$ reduction of TH-positive $(+)$ cells in the ipsilateral substantia nigra pars compacta $(\mathrm{SNc})$ compared to the intact contralateral hemisphere $(10,030 \pm 214$ vs. $1861 \pm 219 ; p<$ 0.001 ; Figure $5 \mathrm{~A}$ ). This was mirrored by an $82.2 \pm 2.0 \%$ reduction in NeuN-positive $(+)$ cells $(10,380 \pm 934$ vs. $1825 \pm 236 ; p<0.001$; Figure $5 \mathrm{~B})$, strongly suggesting 


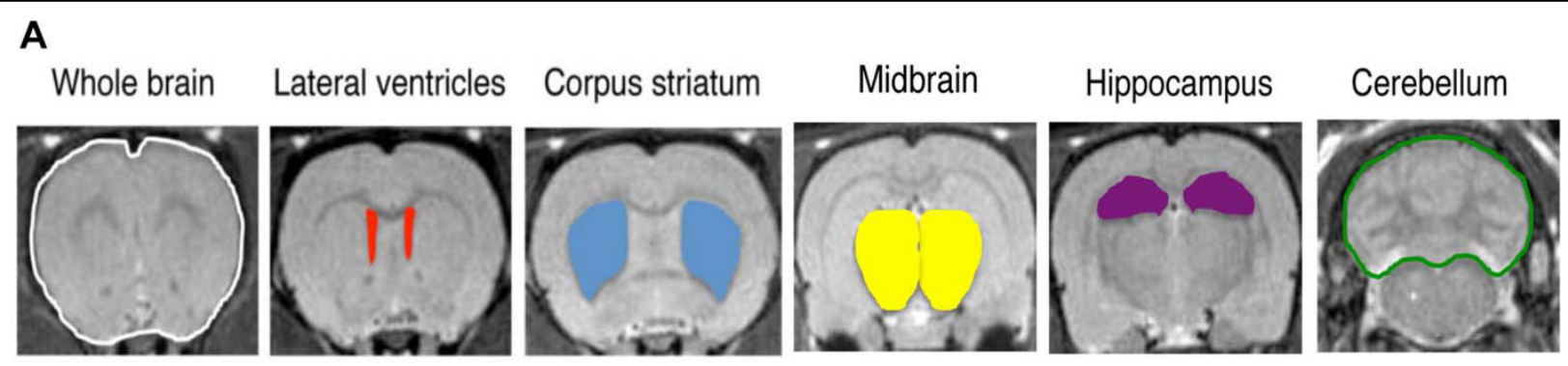

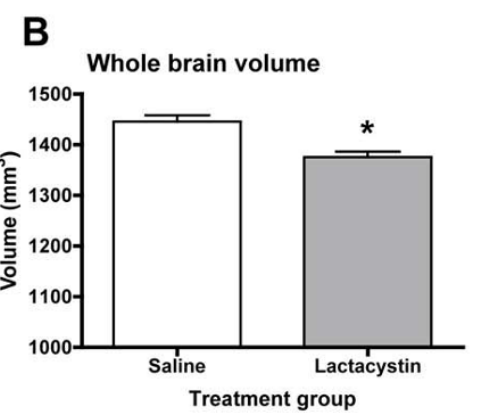

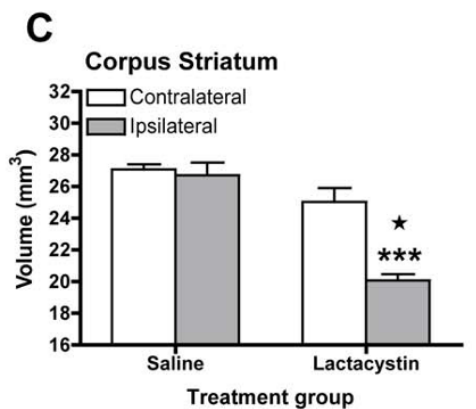

E

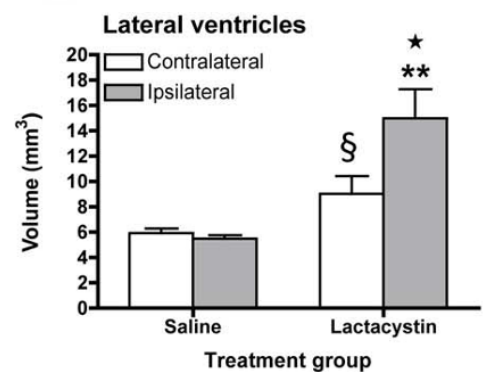

$\mathbf{F}$

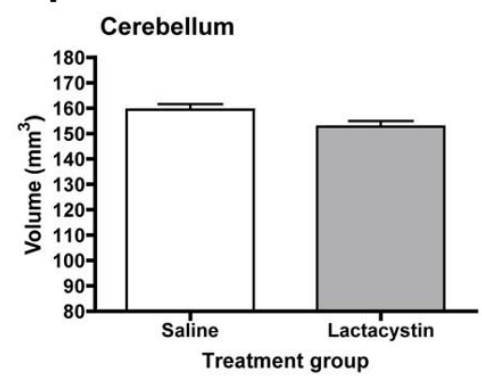

D

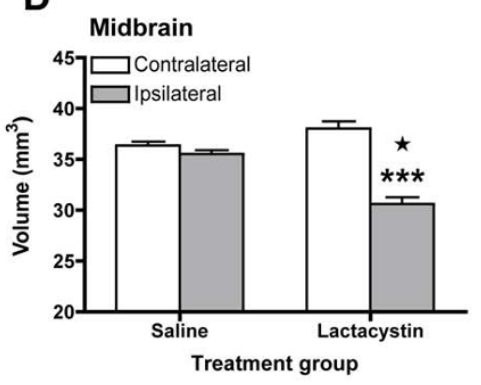

G

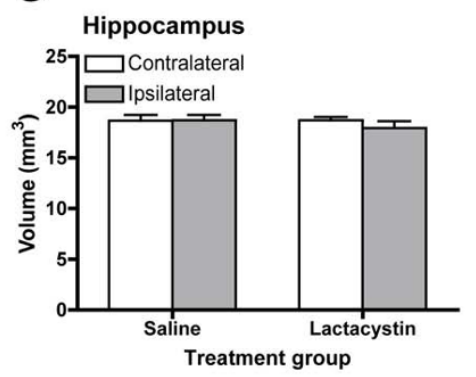

Figure 2 (A) Representative T2W MR images from saline-injected control subject with sample ROls utilised for quantitative volumetric analysis of individual brain regions. (B-G) Bar graphs of regional brain volumetric data measured on in vivo T2W anatomical MRI scans acquired from saline and lactacystin-injected animals 3 weeks post-lesion. (B) Whole brain volume, (C) corpus striatum, (D) ventral midbrain, (E) lateral ventricles, (F) cerebellum and (G) hippocampal formation. Data are expressed as mean volume $\mathrm{mm}^{3} \pm$ s.e.m. ${ }^{*} p<0.05,{ }^{* *} p<0.01$; ipsilateral vs. contralateral hemisphere; ${ }^{*} p<0.01$; ipsilateral hemisphere of lactacystin vs. saline-injected; $\$ p<0.05$ contralateral hemisphere of saline vs. lactacystin-injected.

cell death of A9 DA neurons rather than down-regulation of TH expression in atrophic neurons. No significant differences were observed in either $\mathrm{TH}+$ or $\mathrm{NeuN}+$ cell counts between the contralateral and ipsilateral hemispheres in saline-injected controls or between the contralateral hemispheres in lactacystin-lesioned animals and saline controls (Figure 5A, B). Notably however, the pars reticulata of the $\mathrm{SN}(\mathrm{SNr})$ and the VTA were not free from the direct toxicity of lactacystin. Indeed, qualitatively, extensive loss of both $\mathrm{TH}+$ and $\mathrm{NeuN}+$ cells was observed in these regions when compared to saline controls (Figure 5C, D). Microscopic analysis of extranigral nuclei in the ipsilateral VM revealed additional areas of neuronal loss due to the direct toxicity of lactacystin, including the red nucleus, immediately supranigral to the SNc and in numerous nuclei in the VM adjacent to the needle injection tract. These data are illustrated by representative photomicrographs in Figure 5C and 5D.

Consistent with loss of nigral $\mathrm{TH} / \mathrm{NeuN}+$ cell bodies, densitometry analysis revealed a $69.6 \pm 2.5 \%$ reduction of $\mathrm{TH}+$ fibre density in the ipsilateral striatum, relative to the non-injected contralateral striatum of lactacystinlesioned animals $(61.29 \pm 1.99$ vs. $18.61 \pm 1.54 ; p<$ 0.001; Figure 5E, F) confirming significant loss of DA axon terminals. No significant differences were observed in $\mathrm{TH}+$ fibre optical density in the contralateral striatum when comparing lactacystin-lesioned animals and saline controls, or between the contralateral and ipsilateral striatum in saline-injected animals (Figure 5E, F). 

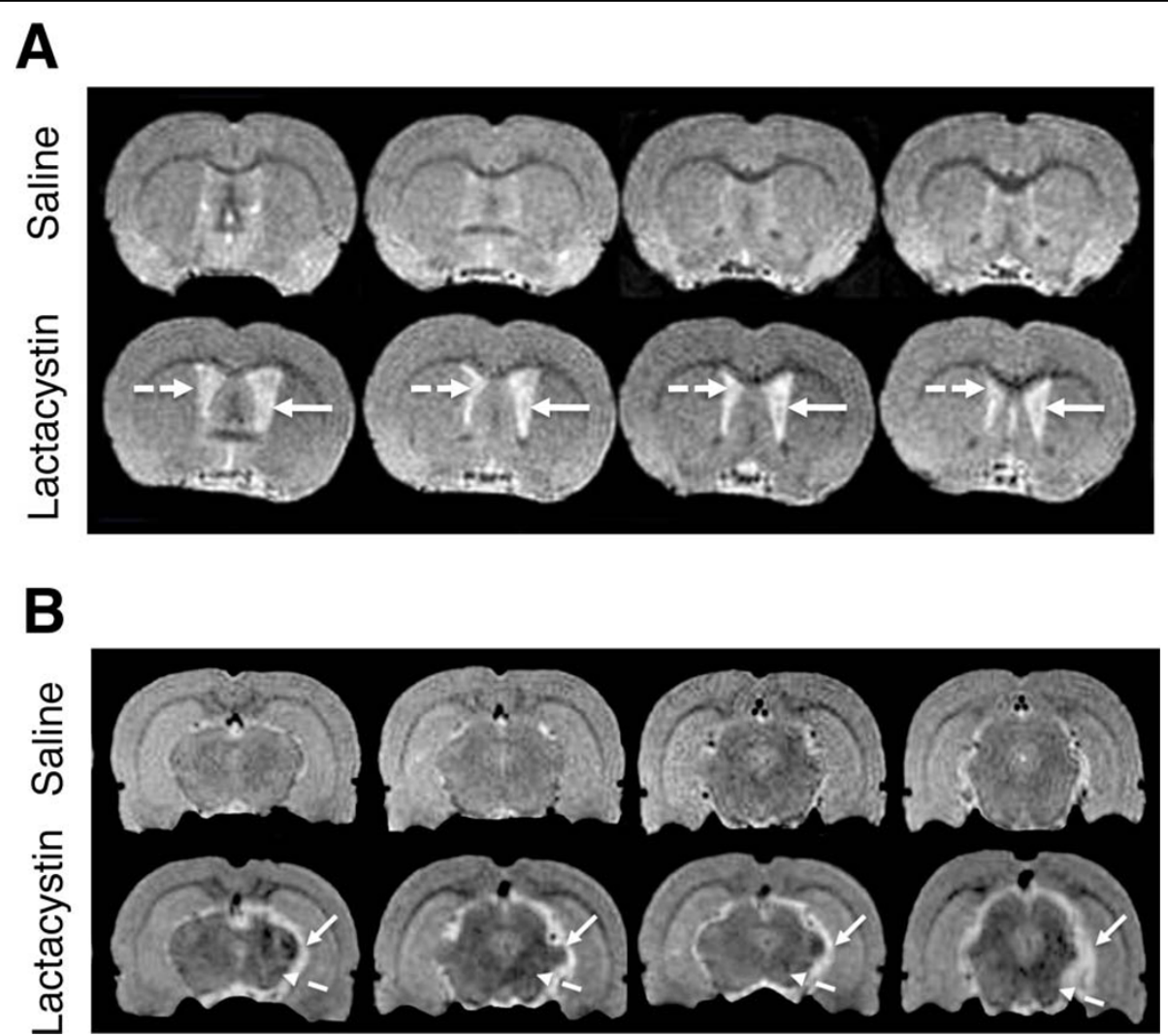

Figure 3 Representative T2W MR images through the striatum (panel A) and midbrain containing the substantia nigra (SN; panel B) to illustrate regional brain atrophy in saline and lactacystin-injected animals. Clear hypertrophy of both the ipsilateral (solid arrows, A) and contralateral (dashed arrows, A) LV can be observed in lactacystin-lesioned animals, compared to saline controls. In the midbrain (panel B) the sub-cortical area containing the SN in the ipsilateral hemisphere appears clearly deformed compared to the intact contralateral hemisphere (solid arrows). Note also the increased CSF signal, suggestive of a volume change in this region. In addition, areas of $T_{2}$ hypointensity are observed in the SN, but also in extra-nigral nuclei (dashed arrows).

Qualitatively, however, there was no apparent loss of either NeuN+ or DARPP-32+ cells in the ipsilateral striatum of lactacystin-lesioned animals, suggesting $\gamma$ amino-butyric-acid (GABA-ergic) medium spiny neurons in the STR may be preserved (Figure 6A,B).

In the SNc of lactacystin-lesioned animals, aggregation of $\alpha$-synuclein was observed, compared to the diffuse staining in the SNc of control animals (Figure 7A-D). Pre-treatment with proteinase $\mathrm{K}$ allowed selective visualisation of insoluble $\alpha$-synuclein deposits and abolished immunostaining for endogenous soluble $\alpha$-synuclein. This revealed focal cytoplasmic proteinase-K resistant $\alpha$-synuclein immunoreactive inclusions in a small proportion of cells in the SNc of lactacystin-lesioned animals, which were not present in saline controls (Figure 7E, F).

Prussian blue (PB) histology revealed minimal iron deposits in the striatum of both lactacystin and salineinjected animals (Figure 8A). By contrast, PB histology revealed clear and extensive iron accumulation in the ipsilateral SNc of lactacystin-lesioned animals compared to saline controls (Figure 8B). In support of this, densitometry analysis exposed a significant increase in iron accumulation in the ipsilateral SNc of lactacystin lesioned animals compared to saline controls (Figure 8C). Iron deposits were, however, also observed in the $\mathrm{SNr}$ and in extra-nigral nuclei in the ventral midbrain, near to the injection tract, as well as in the contralateral hemisphere of lesioned animals compared to saline controls. (Figure 8B, D).

Relationships between in vivo MRI, behaviour and postmortem histology

To determine whether in vivo MRI measurements are linked to behavioural and histological outcomes in this model, we correlated MRI- measurements with behavioural impairments and histological measures of nigrostriatal damage in this model (Tables 2 and 3 , respectively). Significant negative correlations were found between apomorphine rotation and WBV, ipsilateral STR and VM volumes (Table 2), whilst LV hypertrophy correlated positively with apomorphine rotations. By contrast, only ipsilateral STR volume was 


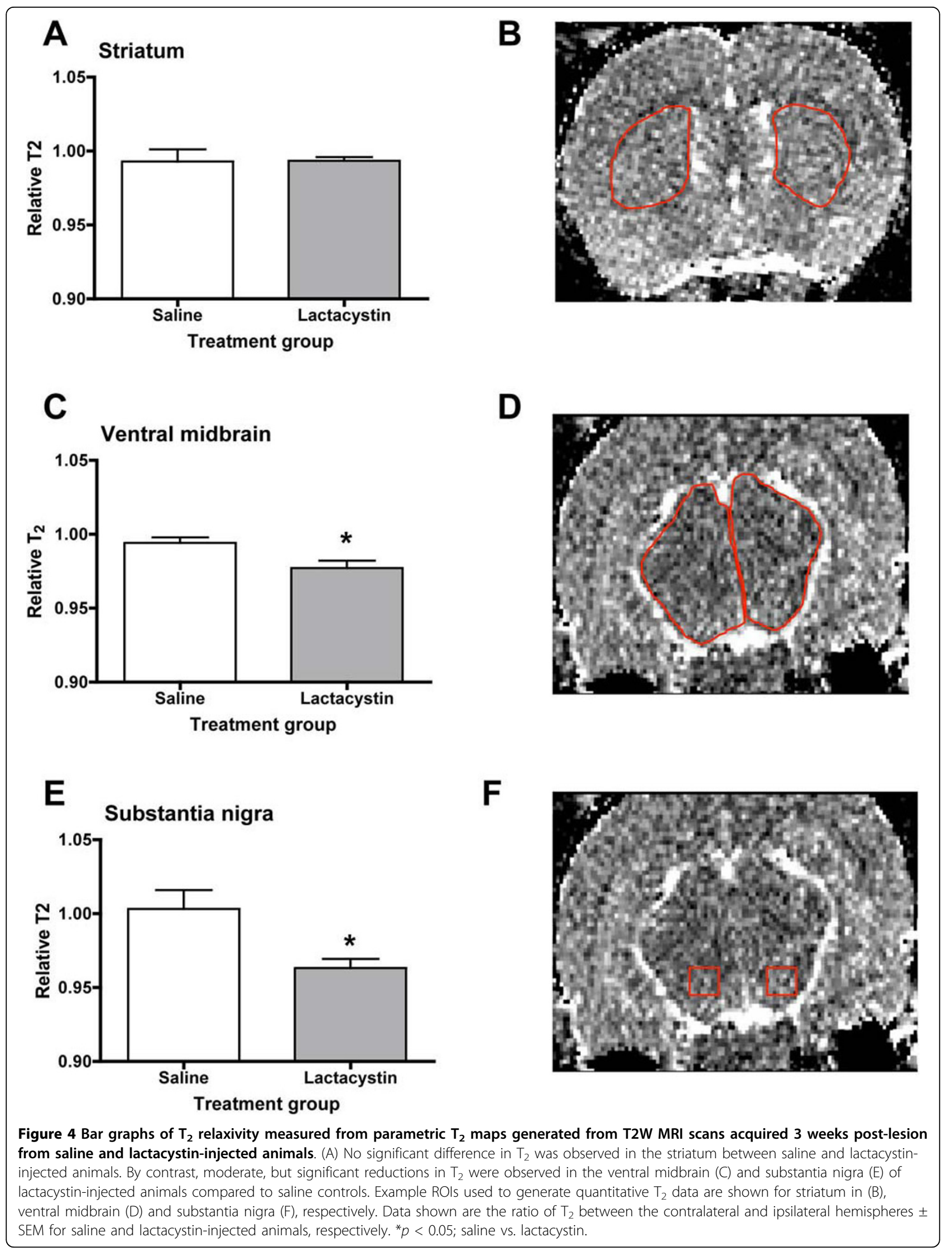




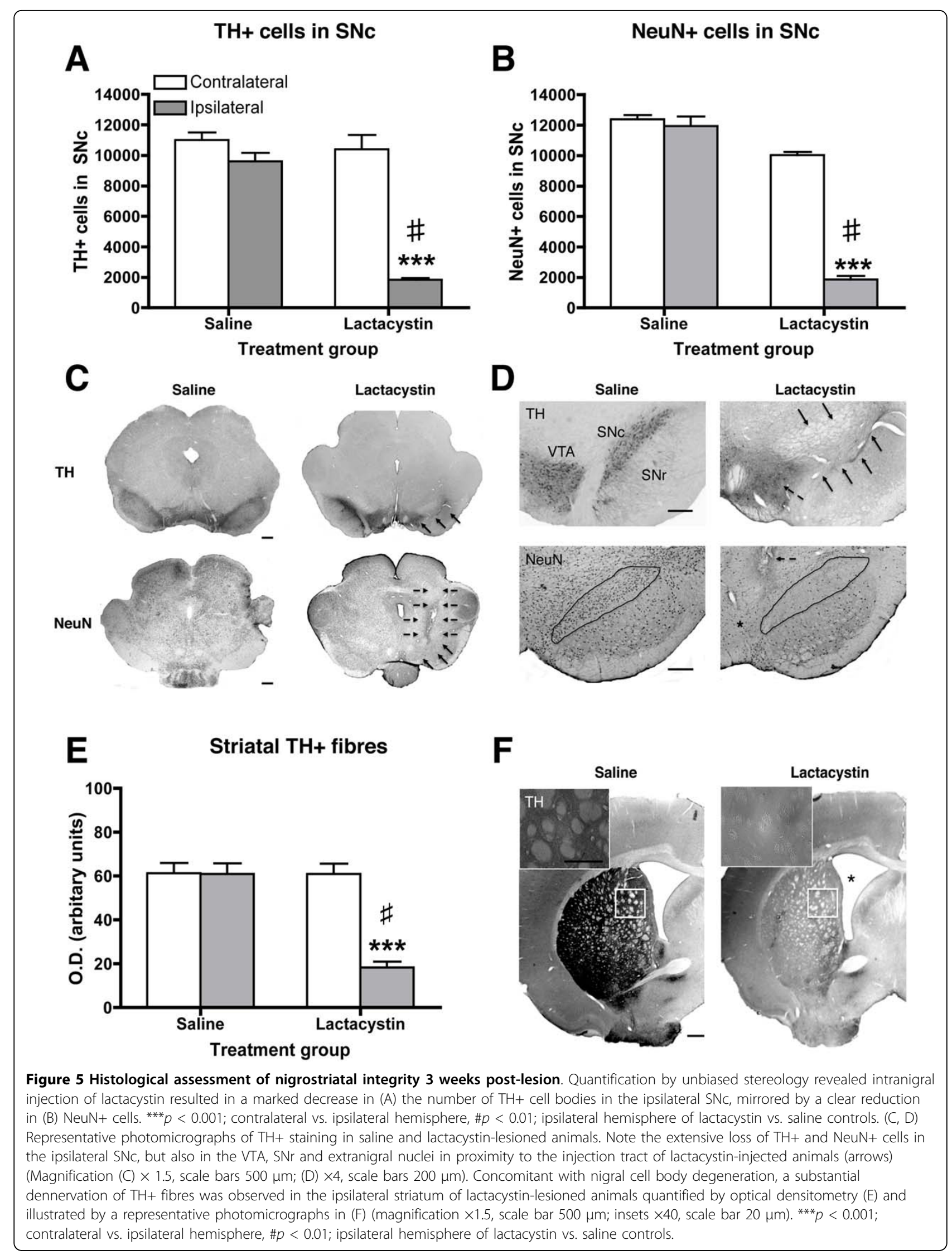




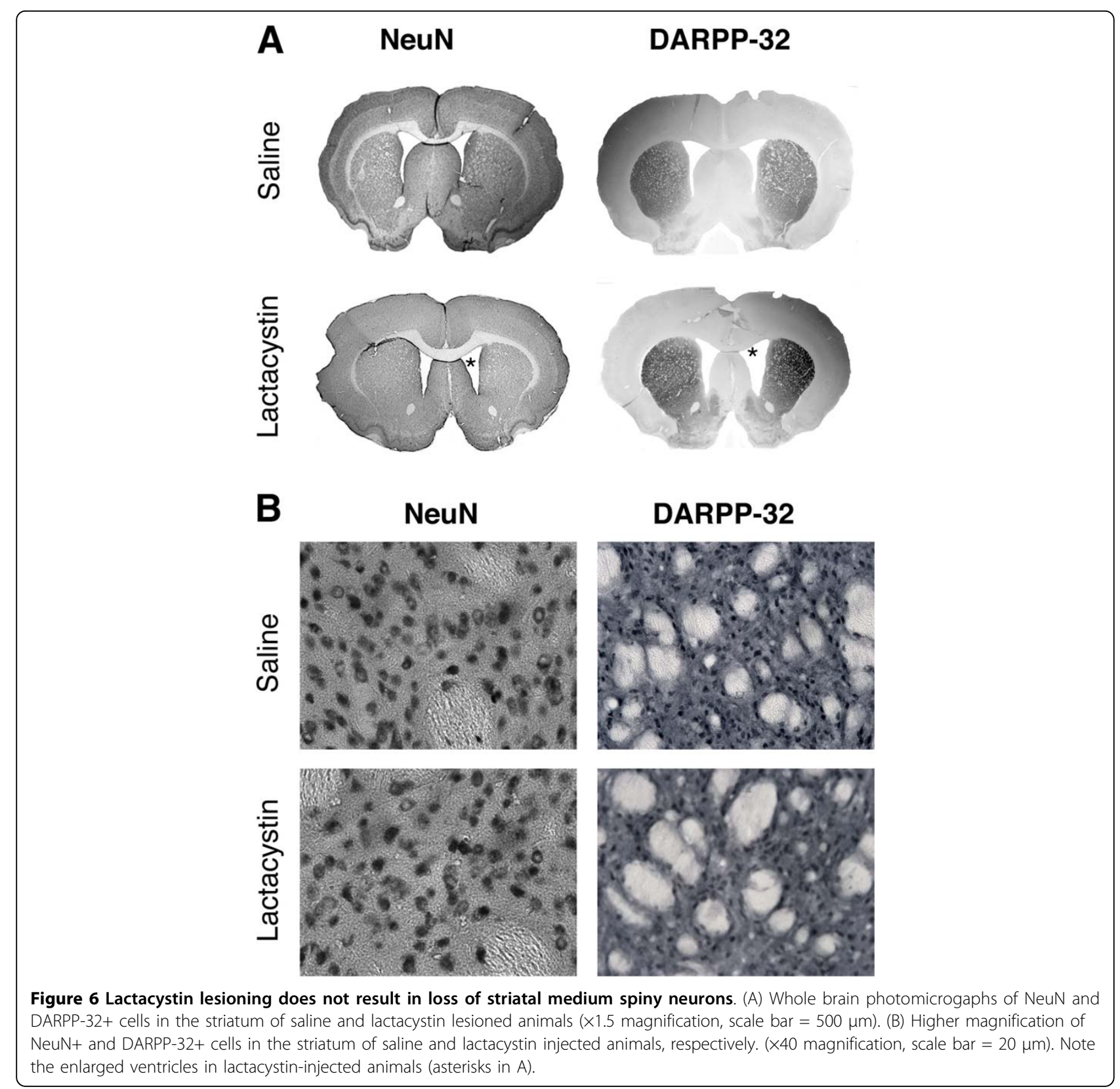

significantly associated with decreased contralateral forelimb grip strength. No significant relationships were observed between either cerebellar volume or hippocampal volume and any behavioural measure. In addition, significant associations were found between the number of $\mathrm{TH}+$ and $\mathrm{NeuN}+$ cells in the ipsilateral SNc with changes in WBV, ipsilateral VM and STR volume. Interestingly, a significant relationship was found between $\mathrm{TH}+$ fibre density and ipsilateral STR volume. In addition, these histological measures all correlated robustly with LV hypertrophy. There was no significant link between any histological measure and HF or CB volume.
No significant associations could be found between ipsilateral $\mathrm{SN} \mathrm{T}_{2}$ values any behavioural measure at 3 weeks post-lesion (Table 3 ). In addition, no significant associations could be found between $\mathrm{SN} \mathrm{T}_{2}$ values and the number of surviving $\mathrm{TH}+$ and $\mathrm{NeuN}+$ cells in the $\mathrm{SN}$, or the density of $\mathrm{TH}+$ fibres in the striatum. However, a significant relationship could be observed between the degree of iron accumulation and $\mathrm{T}_{2}$ values in the SN $(r=-.793 ; p<0.01)$. No significant relationships between striatal $\mathrm{T}_{2}$ values and behavioural or histological outcomes were observed. For completeness, ex vivo post-mortem histological measures were also 


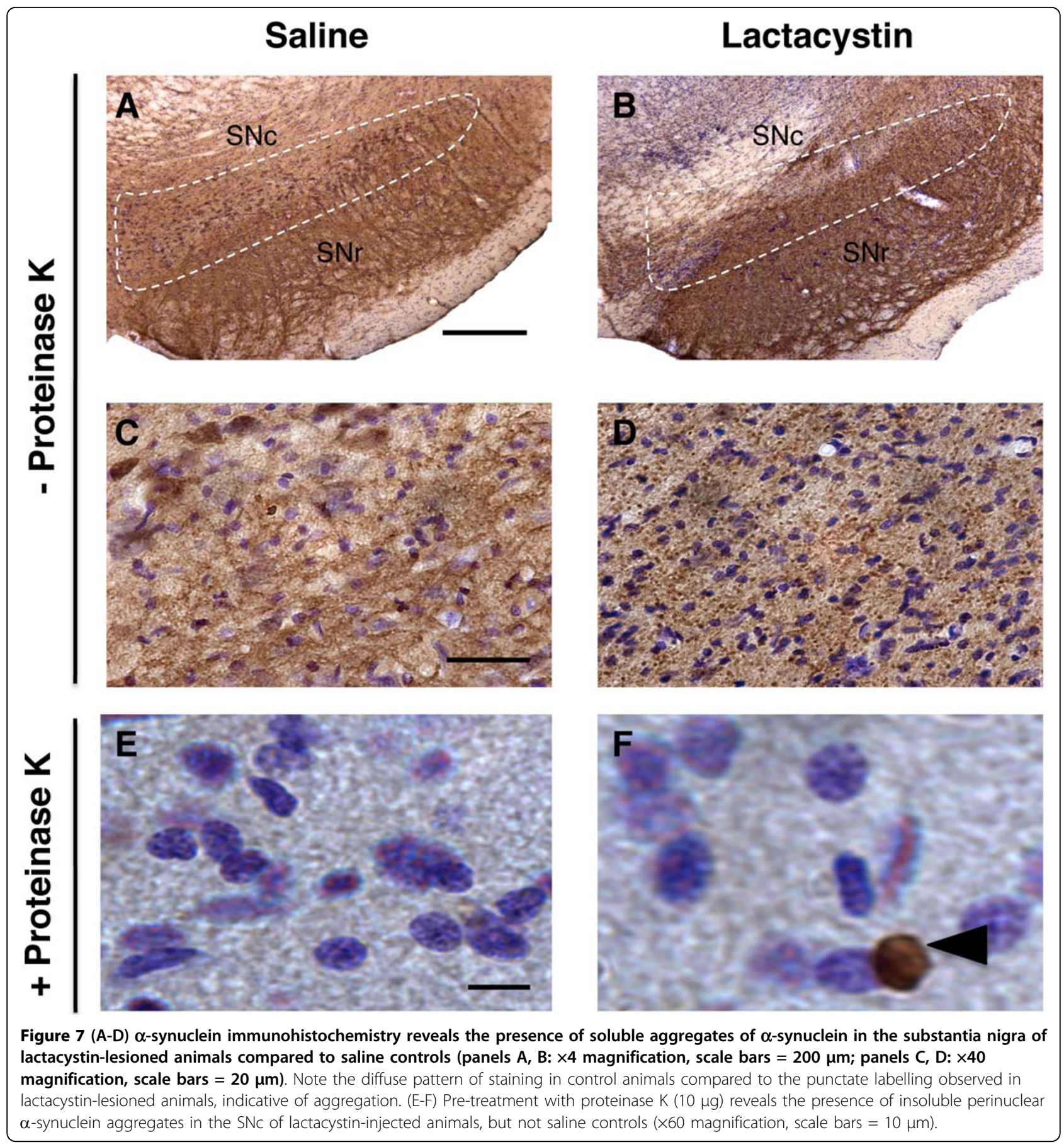

correlated with behavioural outcomes in this model. As expected, there were strong correlations with the number of $\mathrm{TH}+$ cells in the ipsilateral SNc and both apomorphine rotations $(r=-.947 ; p<0.01)$ and forelimb grip strength $(r=.732 ; p<0.05)$. The number of NeuN + cells in the ipsilateral SNc also strongly correlated with both apomorphine rotations $(r=-.882 ; p<0.01)$ and forelimb grip strength $(r=.829 ; p<0.05)$. Similarly, highly significant relationships were observed between
$\mathrm{TH}+$ cell number and NeuN+ cell number in the ipsilateral SNc $(r=.932 ; p<0.01)$ and $\mathrm{TH}+$ fibre density in the striatum $(r=.943 ; p<0.01)$.

\section{Discussion}

Using in vivo T2-weighted (T2W) MRI, we have been able to detect for the first time a pattern of morphological changes in the brains of rodents bearing a unilateral lesion of the nigrostriatal system induced by intranigral 
A
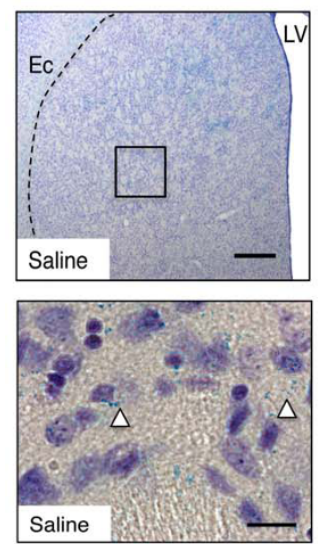

C

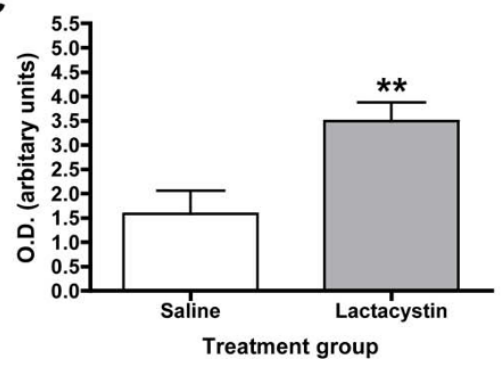

B
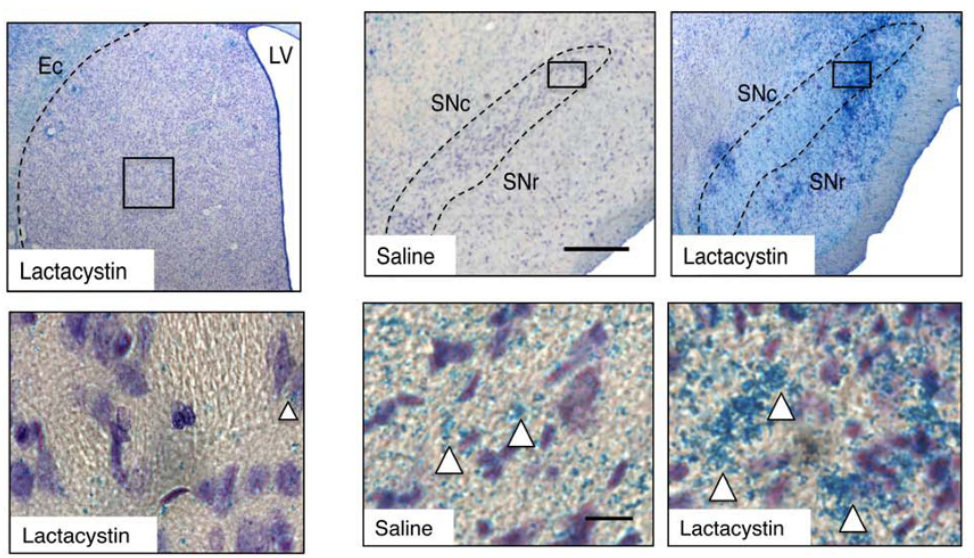

D
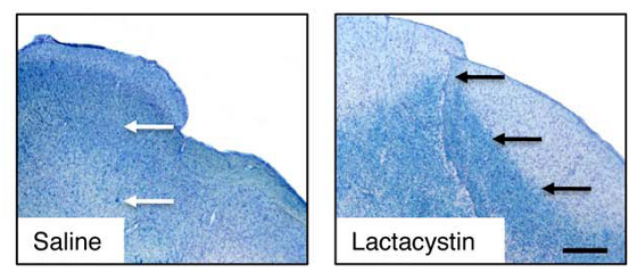

Figure 8 (A) Prussian blue and cresyl violet histology reveals no apparent iron accumulation in the striatum of both saline and lactacystin-lesioned animals (arrowheads in A) ( $\times 2.5$ magnification, scale bars $=300 \mu$ m; insets $\times 40$ magnification, scale bars $=20$ $\boldsymbol{\mu m}$ ). (B) By contrast, extensive iron deposits are observed in the SNc of lactacystin lesioned animals but not saline controls (arrowheads in B); ( $\times 2.5$ magnification, scale bars $=300 \mu \mathrm{m}$; insets $\times 40$ magnification, scale bars $=20 \mu \mathrm{m})$. Note that iron deposition is not confined to the SN but is also present in extra-nigral regions (C) Densitometry measurements confirmed the increased in iron deposition in the SN of lactacystinlesioned animals compared to saline controls. Data shown are mean optical density values (arbitrary units) \pm SEM. ${ }^{* *} p<0.01$; saline vs. lactacystin. (D) Iron deposits were also observed in proximity to the needle-tract of lactacystin-lesioned animals, but not saline controls (arrows in D) $(\times 2.5$ magnification, scale bar $=300 \mu \mathrm{m})$. Interestingly, iron accumulation appears to overlap with areas of neuronal loss in the midbrain of lactacystin-lesioned animals as shown in Figure 5D.

injection of a proteasome inhibitior. We observed a moderate, but significant global reduction in brain volume, accompanied by a striking deformation of the ipsilateral VM, shrinkage of the ipsilateral striatum and hypertrophy of the LV. Although the presence of BG atrophy in PD patients is a matter of controversy [6,7] these data correspond robustly with recent published data from clinical neuroimaging studies in iPD patients.
Indeed, morphological changes, including decreased striatal volume and LV hypertrophy have been reported [4,10-12]. Nevertheless, midbrain atrophy is commonly detected in MRI from patients with atypical PD, such as MSA or PSP $[13,14]$. No changes were observed in either cerebellar or hippocampal volume. We further observed a slight, but significant decrease in $\mathrm{T}_{2}$ relaxivity in the SN, consistent with previous reports of this

Table 2 Two-tailed Pearson correlations for all subjects between specific brain region volumes derived from MRI measurements, behavioural deficits and histological measurements.

\begin{tabular}{cccccc}
\hline Regional MRI volume & Apomorphine rotations & Forelimb grip strength & TH+ cells & NeuN+ cells & TH+ fibres \\
\hline WBV & $-.767^{* *}$ & .420 & $.832^{*}$ & $.718^{*}$ & $.821^{* *}$ \\
VM & $-.955^{* *}$ & .599 & $.980^{* *}$ & $.868^{* *}$ & $.861^{* *}$ \\
STR & $-.746^{* *}$ & $.666^{*}$ & $.798^{*}$ & $.868^{* *}$ & $.715^{* *}$ \\
LV & $.811^{* *}$ & -.467 & $-.775^{*}$ & $-.730^{*}$ & $-.713^{*}$ \\
HPC & .489 & -.211 & -.496 & -.312 & -.398 \\
CB & .025 & -.339 & .081 & -.083 & .034 \\
\hline
\end{tabular}

${ }^{*} p<0.05,{ }^{* *} p<0.01$. 
Table 3 Two-tailed Pearson correlations for all subjects between MRI volume measurements of individual brain regions and whole brain volume. ${ }^{*} p<0.05,{ }^{* *} p<0.01$.

\begin{tabular}{cccccc}
\hline $\mathbf{T}_{\mathbf{2}}$ signal intensity & Apomorphine rotations & Forelimb grip strength & TH+ cells & NeuN+ cells & TH+ fibres \\
\hline SN & -.158 & .405 & .467 & .159 & .452 \\
STR & -.044 & -.076 & .327 & -.341 & -.240 \\
\hline
\end{tabular}

phenomenon in iPD patients [15-18]. However, we could not find any change in $\mathrm{T}_{2}$ in the STR in this model. In addition to these MRI signal changes, lactacystin-lesioned animals displayed robust parkinsonianlike behavioural defects and classical nigrostriatal pathology, including $\alpha$-synuclein aggregation in the $\mathrm{SN}$, consistent with previous reports describing the development of this rodent pre-clinical model [34-36].

Relationship between morphological changes detected by MRI with behavioural and histological outcomes

Examination of the relationship between MRI signal changes and behavioural outcomes revealed that volumetric changes in the ipsilateral VM, striatum and LV were significantly correlated to apomorphine rotation, whilst only ipsilateral striatal volume was significantly associated with impaired grip strength in this model. These data suggest volumetric MRI measurements of these areas could be predictive of the degree of some, but not all, functional impairments in this model, consistent with human studies [4,12]. These preliminary data, albeit in a small number of subjects, raise the possibility that these volumetric in vivo MRI measures may be useful surrogate markers of disease progression in this model. In addition, previous studies have demonstrated that lactacystin-lesioned animals also show significantly impaired performance in the accelerating rotarod test and overall locomotor activity [36]. Thus it may be interesting in future studies to additionally examine the relationship between MRI volumetric changes and performance in these motor tasks in lactacystin-lesioned animals, which may provide further evidence for MRI volumetric changes in this model as surrogate markers of nigrostriatal dysfunction. Importantly, however, repetition of this analysis in a larger group of subjects and comparison to established preclinical models of PD (6-OHDA/MPTP) are required to fully establish the reliability and specificity of these surrogate markers.

In addition, post-mortem validation of MRI signal changes is crucial before these can be accepted as surrogate markers of disease progression. An important corollary to this is that lactacystin can induce both selective damage to DA neurons or more widespread cell loss. Indeed, in vitro, lactacystin has been reported to induce a significant loss of only DA neurons [39] or both DA and GABAergic neurons [40,41]. In contrast, in vivo, intrastriatal injection of lactacystin $(10 \mu \mathrm{g})$ induced a general neuronal toxicity in the striatum [42]. Importantly, in the current study, volume decreases were significantly correlated with the number of surviving $\mathrm{TH}+$ and $\mathrm{NeuN}+$ cell numbers in the ipsilateral $\mathrm{SN}$, but also the density of $\mathrm{TH}+$ fibres in the ipsilateral striatum. However, lactacystin not only induced neuronal loss in the pars compacta and pars reticulata of the $\mathrm{SN}$, but also in the VTA, a nucleus that is relatively preserved in human iPD [1]. Thus, whilst the morphological MRI changes observed in the current study are certainly linked to a specific loss of A9 DA neurons, at least in part, we cannot exclude the possibility that these are also due to an additional loss of other vulnerable neuronal populations.

Additionally, focal areas of neuronal loss were observed throughout the ipsilateral VM along the needle injection tract, consistent with previous reports [42]. This phenomenon most likely explains the extensive deformation of the ipsilateral VM observed on the MRI scans. Thus, although volumetric MRI measures were strongly associated with both behavioural and ex vivo histological measures, their use as surrogate markers of nigrostriatal damage might be limited, since volumetric changes in these regions do not discriminate between damage to the nigrostriatal or other neuronal populations in the midbrain in this model.

Although $\mathrm{TH}+$ fibre density was dramatically reduced in the ipsilateral striatum of lactacystin-lesioned animals, qualitatively, no gross loss of either $\mathrm{NeuN}+$ or DARPP$32+$ cells was apparent. Thus, striatal medium spiny neurons (MSN) appear to be preserved in this model. Interestingly, $\mathrm{TH}+$ fibre loss and dopamine depletion are reported to be associated with synaptic remodelling and dendritic changes in striatal MSN in 6-OHDA lesioned rodents $[43,44]$. These data are consistent with reports of decreased MSN dendrite spine number and length in the striatum of post-mortem PD brains [45]. Conceivably therefore, decreased striatal volume could be attributed to $\mathrm{TH}+$ fibre loss and subsequent dendritic remodelling. It is tempting to speculate therefore that striatal volumetric change may be a conserved marker of nigrostriatal degeneration across pre-clinical models of PD and perhaps human iPD, which importantly, correlates with behavioural and clinical outcomes [4]. However, investigation of volumetric MR changes in established PD models that induce selective nigrostriatal DA cell death, such as 6-OHDA or MPTP, will be important to validate this hypothesis. 
Relationship between $T_{2}$ relaxivity changes detected by MRI with behavioural and histological outcomes In contrast to our findings, previous studies have identified increased $\mathrm{T}_{2}$ relaxation times, visualised as areas of signal hyperintensity on T2W MR images in both 6OHDA-lesioned rodents [27] and MPTP-treated primates [28]. Importantly, these findings were reported at acute time-points post-lesion ( $24 \mathrm{hrs}$ to 7 days) and $\mathrm{T}_{2}$ hyperintense areas were observed to dissipate on subsequent MRI scans with increasing time post-lesion $[27,28]$. It is therefore possible that $T_{2}$ relaxation may evolve with time, although this may not be a reflection of a continuous physiological phenomenon. Indeed, increases in $\mathrm{T}_{2}$ at acute stages post-lesion most likely reflect acute oedema [27]. By contrast, at longer timepoints post-lesion, iron accumulation appears to be the primary cause of $\mathrm{T}_{2}$ signal decrease, as suggested by our findings and those of others [30,46]. Importantly, although previous studies have suggested a link between changes in $\mathrm{SN} \mathrm{T}_{2}$ relaxation time and nigral cell death [27], this has not been robustly established in vivo. We, for instance, could not find a significant relationship between ipsilateral $\mathrm{SN} \mathrm{T}_{2}$ values and either $\mathrm{TH}+$ or $\mathrm{NeuN}+$ cell number in the SNc. Although the number of subjects in our study was small, these data are in agreement with recently published findings in MPTPtreated primates [30]. One plausible explanation of these findings may be related to iron accumulation in the SN. Iron deposition is known to affect MR signal intensity by creating magnetic field inhomogeneities that dephase nearby water-protons leading to shortening of $\mathrm{T}_{2}$ relaxation time and signal drop off in the affected tissues [47]. In support of this, we observed substantial iron accumulation in the ipsilateral SN of lactacystinlesioned animals, but not saline controls. Furthermore, we observed a significant relationship between $T_{2}$ and iron accumulation as measured by Prussian blue histology in the SN 3-weeks post-lesion. These data are in good agreement with the findings of Zhu and colleagues (2007) who initially reported the observation of significant iron accumulation in the $\mathrm{SN}$ in this model by biochemical measurement of iron content in the SN [36]. Interestingly, previous studies have shown that treatment with novel iron chelators prevented nigral iron accumulation and protected the nigrostriatal system against lactacystin-induced neurodegeneration in vivo $[35,36]$. Speculatively therefore, in vivo MR measurement of nigral $T_{2}$ values may be a potentially useful surrogate measure of the neuroprotective efficacy of novel iron chelators in this rodent model of PD, which may warrant further investigation in vivo.

Overall, these data are also consistent with MRI findings in PD patients reporting decreased $\mathrm{T}_{2}$ relaxation time in the SN [15-18] and biochemical measurements reporting increased iron accumulation in the $\mathrm{SN}$ of $\mathrm{PD}$ patients $[48,49]$. Interestingly, changes in $\mathrm{SN} \mathrm{T}_{2}$ relaxation in PD patients appear to correlate with some clinical symptoms, although the exact relationship is unclear $[19,20]$. Based on these clinical reports, it has been suggested that $\mathrm{T}_{2}$ signal changes may be a useful surrogate marker of PD progression $[19,20]$. Importantly, in this $\mathrm{PD}$ model at 3 weeks post-lesion, ipsilateral $\mathrm{SN} \mathrm{T}_{2}$ relaxivity was not significantly associated with any behavioural outcome in this cross-sectional study.

Taken together, it may be suggested that at least two distinct physiological processes underlie changes in nigral $\mathrm{T}_{2}$, which evolve over time and may in part counterbalance each other. Clearly therefore, longitudinal studies are required to further elucidate the relationship between changes in $T_{2}$ and behavioural or histological outcomes in this model to determine whether this can be a useful surrogate marker of ongoing nigrostriatal degeneration in vivo.

It is also noteworthy, however, that in lactacystin lesioned animals we observed numerous areas of $\mathrm{T}_{2}$ hypointensity on our T2W MR images throughout the midbrain and not only in the SN. This finding is inconsistent with the patterns of either iron deposition $[48,49]$ or $T_{2}$ relaxivity changes detected by MRI in PD patients $[19,20]$. This was reflected in both quantitative $\mathrm{T}_{2}$ measurements and post-mortem histology, where clear areas of iron accumulation could be observed not only in the SNc, but also the $\mathrm{SNr}$ as well as in areas surrounding the needle tract, where ongoing neuronal loss was also evident.

\section{Conclusions}

In summary, this study has identified a pattern of morphological changes in lactacystin lesioned animals, as well as alterations in nigral $\mathrm{T}_{2}$ relaxivity, that are somewhat consistent with published clinical neuroimaging data from iPD patients. Interestingly, morphological, but not $\mathrm{T}_{2}$ relaxivity changes, were associated with both behavioural and histological outcomes in this model. This raises the possibility that morphological changes may be non-invasive surrogate markers of nigrostriatal degeneration in vivo in this pre-clinical model. However, post-mortem immunohistological analysis revealed that lactacystin induced non-specific neuronal loss in the VM, as well as widespread iron accumulation. Taken together, these data suggest that MRI signal changes in this PD model are not wholly specific to degeneration of the nigrostriatal system alone, potentially limiting the usefulness of these markers. Nevertheless, this model has pathological relevance to human iPD, and may be a useful tool to study the relationship between proteasome dysfunction, abnormal accumulation of $\alpha$-synuclein and DA neurodegeneration in vivo. Moreover, these data 
reinforce the usefulness of MRI for non-invasive evaluation of pathological changes in the brain in pre-clinical models of neurodegenerative disease.

\section{Methods}

\section{Experimental animals}

Male Sprague-Dawley rats $(250 \pm 10 \mathrm{~g}$, Harlan, UK) were housed in groups of three at $21 \pm 1^{\circ} \mathrm{C}$ on a $12 \mathrm{hr}$ light: dark cycle (lights on 0700, lights off 1900). Standard rat chow and drinking water were available ad libitum. All animal experiments were carried out with local ethical approval and in accordance with the Home Office Animals (Scientific procedures) Act, UK, 1986.

Animals were randomised to either saline or lesion groups ( $n=6$ per group) and anaesthetised by i.p. injection of a mixture of medetomidine hydrochloride (Domitor ${ }^{\ominus} 0.25 \mathrm{mg} / \mathrm{kg}$ ) and ketamine hydrochloride $\left(\right.$ Vetalar $^{\mathrm{TM}}, 230 \mathrm{mg} / \mathrm{kg}$ ) in sterile water and positioned in a stereotaxic frame (Kopf Instruments, Tujunga, CA, USA) with the incisor bar set $3.3 \mathrm{~mm}$ below the interaural line. Nigrostriatal lesions were induced by a unilateral intranigral injection of the proteasome inhibitor lactacystin $(10 \mu \mathrm{g}$ in $2.5 \mu \mathrm{l}$; L6785, Sigma-Aldrich, Poole, UK) at the following stereotaxic co-ordinates, AP: $-5.2 \mathrm{~mm}, \mathrm{ML}:+2.4 \mathrm{~mm}$ lateral from bregma and $-7.6 \mathrm{~mm}$ ventral to dura [50] as previously described [34]. Lactacystin was dissolved in $0.9 \%$ saline $(\mathrm{pH} 7.4)$ immediately prior to use and stored on ice to prevent degradation. Injections were performed at a rate of 1 $\mu \mathrm{l} / \mathrm{min}$ using a motorized syringe pump and the needle was slowly withdrawn 5 min after lesioning to minimize diffusion of toxin into the injection tract. Anesthesia was reversed $1 \mathrm{hr}$ after induction by subcutaneous (s.c.) injection of atipamezole hydrochloride (Antisedan ${ }^{\oplus}, 5 \mathrm{mg} / \mathrm{kg}$ ). Sham-lesioned animals underwent identical surgery, but received an injection of $0.9 \%$ saline. Saline and lactacystin groups were operated on in a randomised fashion in the same surgical session. Post-operative care included analgesia (buphrenorphine, $0.3 \mathrm{mg} / \mathrm{kg}$ s.c. during the first $48 \mathrm{hr}$ ), fluid-replacement ( $4 \mathrm{ml}$ glucosaline solution i.p.) and mashed high-nutrient food pellets during the first week after surgery. Animals were weighed and semiquantitatively scored daily for neurological deficits using a general neurological rating scale as previously described [51]. This was done daily in a blinded fashion until the end of the experiment.

\section{Behavioural testing}

Animals were routinely handled prior to and after surgery in order to calm the animals and enhance reliability when testing. Prior to any behavioural testing (training or trial) animals were allowed to acclimatize to the testing room for $30 \mathrm{~min}$.

\section{Grip strength meter test}

To investigate forelimb motor dysfunction following lactacystin-lesioning performance in the grip strength test was assessed using a grip strength meter (GSM; TSE Systems, Bad Homberg, GER) as previously described [52]. Briefly, forelimb grip strength was tested in sessions consisting of five trials separated by approx. 1 min. Animals underwent training prior to surgery to familiarise themselves with the GSM apparatus. This consisted of one day of testing for five trials, per paw, per animal. Subsequent test sessions were conducted preoperatively to establish a baseline, with subsequent re-testing 3 weeks post-surgery. Test sessions were performed on one day both in the morning and afternoon, as per training with 5 trials per paw, per animal. The mean force exerted on the GSM by each forepaw just prior to grip release was recorded automatically per paw (contralateral and ipsilateral to the lesion) for each animal by a strain gauge connected to a digital readout. An average grip force was then calculated for each limb, for all animals in each group.

\section{Rotational asymmetry}

Three weeks post-lesion, drug-induced rotation as an index of nigrostriatal damage was evaluated in a bank of eight rotameter bowls. Lesioned and control animals were harnessed into jackets tethered to an automated rotameter and allowed to acclimatize for $30 \mathrm{~min}$ before administration of apomorphine hydrochloride $(0.1 \mathrm{mg} / \mathrm{kg}$ dissolved in $0.9 \%$ saline, s.c., Sigma-Aldrich, Poole, UK). The numbers of complete contraversive rotations were then measured over 60 minutes using an automated rotameter (TSE Systems, Bad Homberg, Germany).

\section{Magnetic resonance imaging \\ Preparation}

Animals underwent MRI imaging 3 weeks post-surgery, after completion of behavioural testing, based on previous behavioural and histological studies of nigrostriatal damage in this model [34]. Animals were anesthetized using isoflurane ( $5 \%$ induction, $1.5 \%$ maintenance) in an oxygen/medical air (30\%: 70\%) mixture delivered at $1 \mathrm{~L} /$ min and placed in a PTFE MRI compatible stereotaxic head frame to reduce head movement. Body temperature, respiration rate, blood oxygen saturation and pulse rate were monitored for the duration of the scan. Animal body temperature was maintained at $37^{\circ} \mathrm{C}$ using a heated blanket.

\section{Image acquisition}

T2W MR images were acquired using a 7.0 T horizontal small bore magnet (Varian, Palo Alto, CA, USA) and a custom built head RF coil (David Herlihy) linked to a LINUX-based control console running VnmrJ acquisition software (v2.3, Varian, Palo Alto CA, USA). T2W images were acquired using a multi-echo, multi-slice spin-echo pulse sequence (MEMS), with the following 
scan parameters: FOV $=35 \mathrm{~mm} \times 35 \mathrm{~mm}$; matrix $=192$ $\times 192 ; T R=4200 \mathrm{~ms} ; T E=10,20,30,40,50,60,70,80$ ms; 4 averages, scan duration 54 minutes. Fifty contiguous $500 \mu \mathrm{m}$-thick coronal slices with an in plane resolution of $189 \times 189 \mu \mathrm{m}$ were acquired such that the entire brain of each animal was covered. Once scanning was completed, animals were removed from the magnet bore to a separate holding room, administered $4 \mathrm{ml} 0.9 \%$ saline solution (i.p.) and placed in a heated recovery chamber. Following full recovery from anaesthesia, animals were then returned to their home cages.

\section{Image post-processing}

Brain images were transferred from the console to a workstation, where they were visually inspected prior to post-processing for motion or intensity artefacts. No scans were excluded on this basis. Images were interpolated from the acquired $192 \times 192$ matrix corresponding to a $189 \mu \mathrm{m}$ in-plane linear pixel size to a $256 \times 256$ pixel grid $(136 \mu \mathrm{m}$ voxel size). For quantitative manual volumetric measurements, images corresponding to $\mathrm{TE}$ $=10-80 \mathrm{~ms}$ were summed and converted to Analyse 7.5 (Mayo Clinic). Quantitative $\mathrm{T}_{2}$ relaxation maps were also obtained by a mono-exponential fit of the eight multi-echo images $(\mathrm{TE}=10-80 \mathrm{~ms}$ ) using VnmrJ software.

\section{MR image analysis}

For whole brain and regional volumetric analysis, structures were delineated manually. Individual brain structures were delineated by a single reviewer (A.C.V) blinded to animal surgical status on a slice-by-slice basis in the coronal plane using the ROI tool in JIM v5.0 software (Xinapse systems, Thorpe Waterville, UK). ROIs were traced in both the ipsilateral and contralateral hemispheres of sham and lesioned animals at low magnification followed by manual correction of borders at higher magnification. Clear anatomical landmarks and reference to the standard rodent brain atlas [50] were used to define ROIs for each region analysed in each slice (Table 1). Volumes were calculated by multiplying the sum of the areas of a given structure on all slices by the slice thickness for each animal. Intra-rater-reliability was assessed following repeated measurements using the intra-class-correlation coefficient (ICC). For all brain regions examined, an $I C C$ value of $\geq 0.9$ was obtained, suggesting robustly reliable segmentation of brain structures.

Intensity values for $\mathrm{T}_{2}$ were measured in both the contralateral and ipsilateral hemispheres of saline and lactacystin-lesioned animals in the striatum, ventral midbrain and the $\mathrm{SN}$ from quantitative $\mathrm{T}_{2}$ maps using VnmrJ software. To measure $\mathrm{T}_{2}$, an ROI was drawn using the ROI tool in $\mathrm{VnmrJ}$ on a representative coronal slice, examples of which are shown for each brain region analysed in Figure 4B,D and 4F. A rodent brain atlas
[50] was used to interpret the MR images and define ROI borders. For the SN, atlas plates containing the $\mathrm{SN}$ were overlaid onto MR images to aid identification of this structure. No difference could be made between the pars reticulata and the pars compacta of the $\mathrm{SN}$ thus $\mathrm{T}_{2}$ measurements in this area refer to the whole SN. Values for $\mathrm{T}_{2}$ are expressed as the ratio between the contralateral and ipsilateral striatum and $\mathrm{SN}$ (relative $\mathrm{T}_{2}$ ).

\section{Tissue collection}

One day post-imaging, animals were terminally anaesthetised by a sodium pentobarbital overdose $(60 \mathrm{mg} / \mathrm{kg}$ i.p.) and transcardially perfused first with $0.9 \%$ saline, followed by ice-cold $4 \%$ paraformaldehyde (PFA) in 0.2 $\mathrm{M}$ phosphate buffer, $\mathrm{pH}$ 7.4. Brains were rapidly dissected out, post-fixed for 24 hours and cryo-protected in buffered $30 \%$ sucrose. Serial coronal sections $(40 \mu \mathrm{m})$ were cut on a freezing microtome at $-20^{\circ} \mathrm{C}$ and stored in cryoprotective solution containing $0.05 \%$ sodium azide at $-20^{\circ} \mathrm{C}$ until processed for immunohistochemistry.

\section{Immunohistochemistry}

Free-floating sections were immunostained with the following primary antibodies, rabbit $\alpha$-tyrosine hydroxylase (TH, AB151, Chemicon, 1:3000), mouse $\alpha$-Neuron specific nuclear protein (NeuN, MAB377, Chemicon, $1: 1000)$, mouse anti- $\alpha$-synuclein (610786, BD Biosciences, $1: 100)$ and rabbit $\alpha$-dopamine and cAMP-regulated phosphoprotein-32 (DARPP-32; AB1656; Chemicon, $1: 1000)$ using a standard immunoperoxidase method as described elsewhere [53].

Briefly, sections were rinsed with PBS, then incubated for $10 \mathrm{~min}$ in $0.3 \% \mathrm{H}_{2} \mathrm{O}_{2}$ (Sigma-Aldrich) to quench endogenous peroxidase activity. For $\alpha$-synuclein immunohistochemistry, sections were then incubated with or without PBS containing $10 \mu \mathrm{g} / \mathrm{ml}$ proteinase $\mathrm{K}$ (Promega, Southampton, UK) for $10 \mathrm{~min}$ at room temperature, to visualise both soluble and insoluble $\alpha$-synuclein. Non-specific binding was blocked by incubation for $1 \mathrm{hr}$ in blocking solution containing $10 \%$ normal sera and $0.03 \%$ triton X-100 diluted in PBS. All antibodies were diluted in blocking solution and sections were thoroughly washed in PBS between each step. Tissue sections were incubated with primary antibodies at $4{ }^{\circ} \mathrm{C}$ overnight, followed by $2 \mathrm{hr}$ incubation at room temperature (RT) with secondary biotinylated anti-mouse antibodies (1:200, Vector Laboratories) and a further $1 \mathrm{hr}$ incubation at RT with an avidin-biotinylated-peroxidase complex (Vectastain ABC kit, 1:100 in PBS, Vector Laboratories). The substrate 3,3'-diaminobenzidine (DAB Fast tablets, Sigma-Aldrich, dissolved in $\mathrm{dH}_{2} \mathrm{O}$ with the addition of $\mathrm{H}_{2} \mathrm{O}_{2}$ to a concentration of $0.03 \%$ immediately before use) was used as chromagen. Antibody specificity was confirmed in adjacent tissue sections with the primary or secondary antibody omitted. 
Additional nigral and striatal sections were processed for iron accumulation using Pearl Prussian blue staining as described elsewhere [46]. Finally, sections were washed in PBS, counterstained with cresyl fast violet (CFV) and mounted onto glass slides, dehydrated in graded alcohol and xylene before being coverslipped with DPX (Merck, Lutterworth, UK).

\section{Unbiased stereology cell counts of nigral dopaminergic} neurons

To estimate the remaining number of $\mathrm{TH}$-positive $\mathrm{TH}$ + ) neurons within the SN in sham and lactacystinlesioned animals, cell counts were performed using an unbiased stereological three-level fractionator sampling method on a computerized image analysis set-up (Zeiss Axioscope, Carl Zeiss, Gottingen, GER) running Stereo investigator software (v7.0, MBF Bioscience, Chicago, IL, USA). Every $6^{\text {th }}$ section throughout the rostro-caudal extent of the $\mathrm{SN}$ was systematically sampled using an unbiased counting frame (the "optical fractionator") of known area superimposed on the field of view by the software. In each tissue section analyzed, section thickness was assessed empirically and guard zones of $2 \mu \mathrm{m}$ thickness were used at the top and bottom of each section. The SN was manually outlined at $\times 2.5$ magnification and counting frames were systematically distributed with known $\mathrm{x}$ and $\mathrm{y}$ steps throughout the region from a random starting point. At least 20 counting frames were sampled per section. All cell counts were performed under $\times 40$ magnifications. The coefficients of error (CE) were calculated according to the procedure of West and colleagues with values $<0.10$ accepted [54]. To ensure the absence of bias in cell counting, slides were coded such that the operator was blinded to the surgical status of the animal. To establish that changes in $\mathrm{TH}+$ cells reflected cell death and not reduced expression of $\mathrm{TH}$ protein, additional cell counts were performed in parallel for NeuN-positive $(\mathrm{NeuN}+)$ cells in the $\mathrm{SN}$ as described above.

\section{Optical density measurements}

Optical density of $\mathrm{TH}+$ fibres in the corpus striatum was measured as previously described with modification [55]. Briefly, digital images of TH-stained sections were captured in a blinded fashion from coded slides across 8 different rostral to caudal levels of the striatum $(+1.5$ to $-2.0 \mathrm{~mm}$ AP relative to bregma) at $\times 2.5$ magnification using a Zeiss Axioscope microscope. At each level, for both the ipsilateral and contralateral hemispheres of the brain, images were manually thresholded to highlight $\mathrm{TH}$-staining in the striatum. The area containing the striatum in each section was then traced manually and optical density automatically calculated using the area fraction tool in ImageJ software (NIH, http://rsb.info. nih.gov/ij/). Non-specific background densities were measured in the corpus callosum (an area devoid of $\mathrm{TH}$ staining) and subtracted to give corrected striatal optical density values. This method was also applied to quantify the degree of iron deposition in the SN. Briefly, digital images of tissue sections stained with Prussian blue and nissl stain were captured from coded slides at $\times 40$ magnifications using a Zeiss Axioscope microscope. The optical density of Prussian blue stained cells was quantified as outlined above, from 3-5 sections per subject in each group.

\section{Statistical analysis}

All data are expressed as the mean \pm SEM. Net contraversive turns for lactacystin or saline-injected treated animals in response to apomorphine hydrochloride were plotted against time and the area under the curve (AUC) calculated in Graphpad Prism (v4.0 San Diego, $\mathrm{CA}, \mathrm{USA})$. The resultant means of AUC \pm SEM for lactacystin or saline-injected animals were then compared using two-tailed paired student's t-test. Grip strength data, $M R$ regional volumes, quantitative $T_{2}$ measurements and histological data were analysed using standard paired two-tailed student t-tests to compare differences within groups and unpaired two-tailed t-tests to compare differences between groups in Graphpad Prism. Correlations between regional brain volume changes, histology and motor behaviour were calculated for all subjects (both saline controls and lactacystinlesioned animals) using a two-tailed Pearson correlation analysis in SPSS (v16.0, Woking, UK). In all cases, statistical significance was set at $p<0.05$.

\section{Acknowledgements}

This study was supported by a grant from the Edmond J. Safra philanthropic foundation, which we thank for their generous financial assistance. We also thank the British Heart Foundation for supporting the $7 \mathrm{~T}$ MRI scanner (Preclinical imaging unit, Kings College London) used in this study. ACV is supported by an Edmond J Safra fellowship. MM is supported by an RCUK Fellowship.

\section{Authors' contributions}

ACV participated in the design of the study, carried out in vivo MR imaging, MR image analysis, behavioural testing, immunohistochemistry, stereology cell counting, optical density measurements, statistical analysis and drafted the manuscript. SJ assisted with surgery, stereology, cell counting, and optical density measurements and helped to draft the manuscript. MM conceived of the study, participated in its design and helped to draft the manuscript. All authors read and approved the final manuscript.

Received: 16 July 2009

Accepted: 5 January 2010 Published: 5 January 2010

\section{References}

1. Fearnley JM, Lees AJ: Ageing and Parkinson's disease: substantia nigra regional selectivity. Brain 1991, 114(Pt 5):2283-2301.

2. Spillantini MG, Schmidt ML, Lee VM, Trojanowski JQ, Jakes $R$, Goedert M: Alpha-synuclein in Lewy bodies. Nature 1997, 388(6645):839-840. 
3. Brooks $D$ J: The role of structural and functional imaging in parkinsonian states with a description of PET technology. Semin Neurol 2008, 28(4):435445.

4. Reetz K, Gaser C, Klein C, Hagenah J, Buchel C, Gottschalk S, Pramstaller PP, Siebner HR, Binkofski F: Structural findings in the basal ganglia in genetically determined and idiopathic Parkinson's disease. Mov Disord 2009, 24(1):99-103.

5. Aarsland D, Perry R, Brown A, Larsen JP, Ballard C: Neuropathology of dementia in Parkinson's disease: a prospective, community-based study. Ann Neurol 2005, 58(5):773-776.

6. Burton EJ, McKeith IG, O'Burn DJ, Brien JT: Brain atrophy rates in Parkinson's disease with and without dementia using serial magnetic resonance imaging. Mov Disord 2005, 20(12):1571-1576.

7. Ghaemi M, Hilker R, Rudolf J, Sobesky J, Heiss WD: Differentiating multiple system atrophy from Parkinson's disease: contribution of striatal and midbrain MRI volumetry and multi-tracer PET imaging. I Neurol Neurosurg Psychiatry 2002, 73(5):517-523.

8. Alegret M, Junque C, Pueyo R, Valldeoriola F, Vendrell P, Tolosa E, Mercader JM: MRI atrophy parameters related to cognitive and motor impairment in Parkinson's disease. Neurologia 2001, 16(2):63-69.

9. Brenneis C, Seppi K, Schocke MF, Muller J, Luginger E, Bosch S, Loscher WN, Buchel C, Poewe W, Wenning GK: Voxel-based morphometry detects cortical atrophy in the Parkinson variant of multiple system atrophy. Mov Disord 2003, 18(10):1132-1138.

10. Krabbe K, Karlsborg M, Hansen A, Werdelin L, Mehlsen J, Larsson HB, Paulson OB: Increased intracranial volume in Parkinson's disease. J Neurol Sci 2005, 239(1):45-52.

11. Camicioli R, Gee M, Bouchard TP, Fisher NJ, Hanstock CC, Emery DJ, Martin WR: Voxel-based morphometry reveals extra-nigral atrophy patterns associated with dopamine refractory cognitive and motor impairment in parkinsonism. Parkinsonism Relat Disord 2009, 15(3):187-195.

12. Lewis MM, Smith AB, Styner M, Gu H, Poole R, Zhu H, Li Y, Barbero X, Gouttard S, McKeown MJ, et al: Asymmetrical lateral ventricular enlargement in Parkinson's disease. Eur J Neurol 2009, 16(4):475-481

13. Paviour DC, Price SL, Jahanshahi M, Lees AJ, Fox NC: Regional brain volumes distinguish PSP, MSA-P, and PD: MRI-based clinico-radiological correlations. Mov Disord 2006, 21(7):989-996.

14. Paviour DC, Price SL, Jahanshahi M, Lees AJ, Fox NC: Longitudinal MRI in progressive supranuclear palsy and multiple system atrophy: rates and regions of atrophy. Brain 2006, 129(Pt 4):1040-1049.

15. Antonini A, Leenders KL, Meier D, Oertel WH, Boesiger P, Anliker M: T2 relaxation time in patients with Parkinson's disease. Neurology 1993, 43(4):697-700.

16. Brar S, Henderson D, Schenck J, Zimmerman EA: Iron accumulation in the substantia nigra of patients with Alzheimer disease and parkinsonism. Arch Neurol 2009, 66(3):371-374.

17. Kosta P, Argyropoulou MI, Markoula S, Konitsiotis S: MRI evaluation of the basal ganglia size and iron content in patients with Parkinson's disease. J Neurol 2006, 253(1):26-32.

18. Vymazal J, Righini A, Brooks RA, Canesi M, Mariani C, Leonardi M, Pezzoli G: $\mathrm{T} 1$ and $\mathrm{T} 2$ in the brain of healthy subjects, patients with Parkinson disease, and patients with multiple system atrophy: relation to iron content. Radiology 1999, 211(2):489-495.

19. Martin WR, Wieler M, Gee M: Midbrain iron content in early Parkinson disease: a potential biomarker of disease status. Neurology 2008, 70(16 Pt 2):1411-1417.

20. Wallis LI, Paley MN, Graham JM, Grunewald RA, Wignall EL, Joy HM, Griffiths PD: MRI assessment of basal ganglia iron deposition in Parkinson's disease. J Magn Reson Imaging 2008, 28(5):1061-1067.

21. Pavese N, Brooks DJ: Imaging neurodegeneration in Parkinson's disease. Biochim Biophys Acta 2009, 1792(7):722-729.

22. Jenner P: Functional models of Parkinson's disease: a valuable tool in the development of novel therapies. Ann Neurol 2008, 64(Suppl 2):S16-29.

23. Lythgoe MF, Sibson NR, Harris NG: Neuroimaging of animal models of brain disease. Br Med Bull 2003, 65:235-257.

24. Sanchez-Pernaute $R$, Brownell AL, Jenkins BG, Isacson O: Insights into Parkinson's disease models and neurotoxicity using non-invasive imaging. Toxicol Appl Pharmacol 2005, 207(2 Suppl):251-256.

25. Ashioti M, Beech JS, Lowe AS, Hesselink MB, Modo M, Williams SC: Multimodal characterisation of the neocortical clip model of focal cerebral ischaemia by MRI, behaviour and immunohistochemistry. Brain Res 2007, 1145:177-189.

26. Guzman R, Lovblad KO, Meyer M, Spenger C, Schroth G, Widmer HR: Imaging the rat brain on a $1.5 \mathrm{~T}$ clinical MR-scanner. J Neurosci Methods 2000, 97(1):77-85.

27. Kondoh T, Bannai M, Nishino H, Torii K: 6-Hydroxydopamine-induced lesions in a rat model of hemi-Parkinson's disease monitored by magnetic resonance imaging. Exp Neurol 2005, 192(1):194-202.

28. Miletich RS, Bankiewicz KS, Quarantelli M, Plunkett RJ, Frank J, Kopin IJ, Di Chiro G: MRI detects acute degeneration of the nigrostriatal dopamine system after MPTP exposure in hemiparkinsonian monkeys. Ann Neurol 1994, 35(6):689-697.

29. Podell M, Hadjiconstantinou M, Smith MA, Neff NH: Proton magnetic resonance imaging and spectroscopy identify metabolic changes in the striatum in the MPTP feline model of parkinsonism. Exp Neurol 2003, 179(2):159-166.

30. van Vliet SA, Blezer EL, Jongsma MJ, Vanwersch RA, Olivier B, Philippens $I H$ : Exploring the neuroprotective effects of modafinil in a marmoset Parkinson model with immunohistochemistry, magnetic resonance imaging and spectroscopy. Brain Res 2008, 1189:219-228.

31. Zhang Z, Zhang M, Ai Y, Avison C, Gash DM: MPTP-Induced pallidal lesions in rhesus monkeys. Exp Neurol 1999, 155(1):140-149.

32. Boska MD, Hasan KM, Kibuule D, Banerjee R, McIntyre E, Nelson JA, Hahn T, Gendelman HE, Mosley RL: Quantitative diffusion tensor imaging detects dopaminergic neuronal degeneration in a murine model of Parkinson's disease. Neurobiol Dis 2007, 26(3):590-596.

33. Pelled G, Bergman H, Ben-Hur T, Goelman G: Manganese-enhanced MRI in a rat model of Parkinson's disease. J Magn Reson Imaging 2007, 26(4):863870.

34. McNaught KS, Bjorklund LM, Belizaire R, Isacson O, Jenner P, Olanow CW: Proteasome inhibition causes nigral degeneration with inclusion bodies in rats. Neuroreport 2002, 13(11):1437-1441.

35. Zhang X, Xie W, Qu S, Pan T, Wang X, Le W: Neuroprotection by iron chelator against proteasome inhibitor-induced nigral degeneration. Biochem Biophys Res Commun 2005, 333(2):544-549.

36. Zhu W, Xie W, Pan T, Xu P, Fridkin M, Zheng H, Jankovic J, Youdim MB, Le W: Prevention and restoration of lactacystin-induced nigrostriatal dopamine neuron degeneration by novel brain-permeable iron chelators. Faseb J 2007, 21(14):3835-3844.

37. Pan T, Kondo S, Zhu W, Xie W, Jankovic J, Le W: Neuroprotection of rapamycin in lactacystin-induced neurodegeneration via autophagy enhancement. Neurobiol Dis 2008, 32(1):16-25.

38. Zhu W, Xie W, Pan T, Jankovic J, Li J, Youdim MB, Le W: Comparison of neuroprotective and neurorestorative capabilities of rasagiline and selegiline against lactacystin-induced nigrostriatal dopaminergic degeneration. J Neurochem 2008, 105(5):1970-1978.

39. McNaught KS, Mytilineou C, Jnobaptiste R, Yabut J, Shashidharan P, Jennert $P$, Olanow CW: Impairment of the ubiquitin-proteasome system causes dopaminergic cell death and inclusion body formation in ventral mesencephalic cultures. J Neurochem 2002, 81(2):301-306.

40. Mathur BN, Neely MD, Dyllick-Brenzinger M, Tandon A, Deutch AY: Systemic administration of a proteasome inhibitor does not cause nigrostriatal dopamine degeneration. Brain Res 2007, 1168:83-89.

41. Reaney SH, Johnston LC, Langston WJ, Di Monte DA: Comparison of the neurotoxic effects of proteasomal inhibitors in primary mesencephalic cultures. Exp Neurol 2006, 202(2):434-440.

42. Miwa H, Kubo T, Suzuki A, Nishi K, Kondo T: Retrograde dopaminergic neuron degeneration following intrastriatal proteasome inhibition. Neurosci Lett 2005, 380(1-2):93-98.

43. Day $M$, Wang Z, Ding J, An X, Ingham CA, Shering AF, Wokosin D, lijic E, Sun Z, Sampson AR, et al: Selective elimination of glutamatergic synapses on striatopallidal neurons in Parkinson disease models. Nat Neurosci 2006, 9(2):251-259.

44. Solis $\mathrm{O}$, Limon DI, Flores-Hernandez J, Flores G: Alterations in dendritic morphology of the prefrontal cortical and striatum neurons in the unilateral 6-OHDA-rat model of Parkinson's disease. Synapse (New York, NY) 2007, 61(6):450-458.

45. Deutch AY, Colbran RJ, Winder DJ: Striatal plasticity and medium spiny neuron dendritic remodeling in parkinsonism. Parkinsonism Relat Disord 2007, 13(Suppl 3):S251-258. 
46. Justicia C, Ramos-Cabrer P, Hoehn M: MRI detection of secondary damage after stroke: chronic iron accumulation in the thalamus of the rat brain. Stroke 2008, 39(5):1541-1547.

47. Chen JC, Hardy PA, Kucharczyk W, Clauberg M, Joshi JG, Vourlas A, Dhar M, Henkelman RM: MR of human postmortem brain tissue: correlative study between T2 and assays of iron and ferritin in Parkinson and Huntington disease. AJNR Am J Neuroradiol 1993, 14(2):275-281.

48. Dexter DT, Wells FR, Lees AJ, Agid F, Agid Y, Jenner P, Marsden CD: Increased nigral iron content and alterations in other metal ions occurring in brain in Parkinson's disease. J Neurochem 1989, 52(6):18301836.

49. Griffiths PD, Dobson BR, Jones GR, Clarke DT: Iron in the basal ganglia in Parkinson's disease. An in vitro study using extended X-ray absorption fine structure and cryo-electron microscopy. Brain 1999, 122(Pt 4):667673.

50. Paxinos $G$, Watson $C$ : The rat brain in stereotaxic co-ordinates Academic Press, 62007.

51. Modo M, Stroemer RP, Tang E, Veizovic T, Sowniski P, Hodges $H$ : Neurological sequelae and long-term behavioural assessment of rats with transient middle cerebral artery occlusion. J Neurosci Methods 2000 104(1):99-109.

52. Dunnett SB, Carter RJ, Watts C, Torres EM, Mahal A, Mangiarini L, Bates G, Morton AJ: Striatal transplantation in a transgenic mouse model of Huntington's disease. Exp Neurol 1998, 154(1):31-40.

53. Vernon AC, Zbarsky V, Datla KP, Croucher MJ, Dexter DT: Subtype selective antagonism of substantia nigra pars compacta Group I metabotropic glutamate receptors protects the nigrostriatal system against 6hydroxydopamine toxicity in vivo. J Neurochem 2007, 103(3):1075-1091.

54. West MJ, Slomianka L, Gundersen HJ: Unbiased stereological estimation of the total number of neurons in thesubdivisions of the rat hippocampus using the optical fractionator. Anat Rec 1991, 231(4):482-497.

55. Debeir T, Ginestet L, Francois C, Laurens S, Martel JC, Chopin P, Marien M, Colpaert F, Raisman-Vozari R: Effect of intrastriatal 6-OHDA lesion on dopaminergic innervation of the rat cortex and globus pallidus. Exp Neurol 2005, 193(2):444-454.

doi:10.1186/1471-2202-11-1

Cite this article as: Vernon et al:: Non-invasive evaluation of nigrostriatal neuropathology in a proteasome inhibitor rodent model of Parkinson's disease. BMC Neuroscience 2010 11:1.

Publish with Bio Med Central and every scientist can read your work free of charge

"BioMed Central will be the most significant development for disseminating the results of biomedical research in our lifetime. "

Sir Paul Nurse, Cancer Research UK

Your research papers will be:

- available free of charge to the entire biomedical community

- peer reviewed and published immediately upon acceptance

- cited in PubMed and archived on PubMed Central

- yours - you keep the copyright 